\title{
TRATAMENTO ENDODÔNTICO EM DENS IN DENTE: REVISÃO DE LITERATURA
}

\section{ENDODONTIC TREATMENT OF DENS IN DENTE: LITERATURE REVIEW}

\author{
Karina Soares Hidalgo $^{1}$ (D), Melissa Ayumi Tateyama ${ }^{2}$ (D), Marcos Sérgio Endo ${ }^{1 *}$ (D) \\ ${ }^{1}$ UNINGÁ - Centro Universitário Ingá, Maringá, PR, Brasil. \\ ${ }^{2}$ Universidade Estadual de Maringá, Maringá, PR, Brasil. \\ *marcossendo@gmail.com
}

\section{RESUMO}

Dens in dente é uma anormalidade descrita como o dobramento do órgão do esmalte na papila dentária durante o estágio de formação. Acomete principalmente os incisivos laterais superiores, sendo sua frequência de ocorrência de $0,04 \%$ a $10 \%$. O objetivo deste trabalho é apresentar as diferentes intervenções clínicas descritas na literatura com foco na necessidade de tratamento endodôntico em casos de dens in dente. Foi realizada uma busca nas bases de dados PubMed, Biblioteca Virtual da Saúde (BVS) e Google Acadêmico utilizando as palavras-chave: "dens in dente", "dens invaginatus", "dental malformation", "endodontic treatment", "root canal treatment", "endodontic therapy", "endodontics" combinadas com os operadores booleanos "AND" e "OR". O tratamento do dens in dente inclui o selamento de esmalte, tratamento endodôntico, tratamento cirúrgico combinado, reimplante intencional e extração. A escolha do tratamento depende de sua classificação, que pode ser dividido em tipo I, II ou III. O uso da tomografia tem sido indicado para diagnóstico e planejamento. A limpeza e a modelagem nesses casos se mostram de difícil complexidade. O uso de insertos ultrassônicos podem ser utilizados para potencializar os efeitos da substância química auxiliar na limpeza do canal. A técnica de obturação termoplastificada se mostra satisfatória por proporcionar um melhor preenchimento na obturação do canal radicular. Conclui-se que para a abordagem dos casos de Dens in dente, o profissional necessita ter conhecimento sobre diagnóstico, anatomia interna, interpretação radiográfica e tomográfica, e conhecer as possibilidades de intervenção clínica de acordo com a sua classificação.

Palavras-chave: Dens in Dente. Dens Invaginatus. Malformação Dentária. Tratamento Endodôntico.

\section{ABSTRACT}

Dens in dens is an abnormality described as a bending of the enamel organ into the dental papilla during the tooth development stage. It affects especially the upper lateral incisors in a frequency of $0.04 \%-10 \%$. The objective of this study was to discuss the different clinical interventions in the literature and focusing on the need for endodontic treatment in cases of dens in dens. A search in the databases PubMed, Biblioteca Virtual da Saúde (BVS) and Google Scholar was carried out using the keywords: "dens in dente", "dens invaginatus", "dental malformation", "endodontic treatment", "root canal treatment", "endodontic therapy", "endodontics" combined with the Boolean operators "AND" and "OR". Dens in dens treatments include enamel sealing, endodontic treatment, combined surgical treatment, intentional reimplantation, and extraction. The treatment is selected according to the classification, which can be divided into type I, II or III. Tomography has been indicated for diagnosis and planning. Cleaning and modeling, in these cases, are difficult and complex. Ultrasonic inserts can be used to enhance the effects of the auxiliary chemical substance in cleaning the canal. The thermoplasticized gutta-percha filling is satisfactory by providing a better filling in the root canal obturation. It can be concluded that to treat the cases of Dens in dens, the dentist needs to have knowledge about diagnosis, anatomy, radiographic and tomographic interpretation, and to know the possibilities of clinical intervention according to its classification. Keywords: Dens in Dens. Dens Invaginatus. Dental Malformation. Endodontic Treatment. 


\section{INTRODUÇÃO}

Dens in dente ou dens invaginatus é a designação dada a uma rara anomalia dentária (KIRZIOGLU; CEYHAN, 2009) que ocorre na fase da odontogênese, em que o desenvolvimento do órgão do esmalte é defeituoso, resultando numa morfodiferenciação caracterizada pela invaginação do esmalte na borda incisal (SERRANO, 1991; KARACA; TOLLER, 1992; SKONER; WALLACE, 1994). Essa malformação interna pode ser exclusiva à câmara pulpar, estender-se até a raiz ou, em casos extremos, ao ápice (HULSMANN, 1997). A etiologia do dens in dente ainda é desconhecida, embora existam algumas evidências de que pode ser de origem genética (ALANI; BISHOP, 2008; ISHIDA et al., 2016; RANGANATHAN, SUNDARESAN e RAMASAMY, 2016). Na maioria dos casos, pode ser diagnosticado em um atendimento odontológico rotineiro em exame radiográfico, tendo a aparência de um dente dentro do outro (SOUZA; BRAMANTE, 1998) e, clinicamente, pode apresentar assimetria incomum da coroa; porém, em alguns casos, a LOCAcoroa pode apresentar-se em aspecto de normalidade (HULSMANN, 1997).

Possui uma prevalência de 0,04\% a 10\% (ALANI; BISHOP, 2008; NEVILLE et al., 2009) e pode acometer tanto a dentição decídua (NEVILLE et al., 2009) quanto a permanente, tendo predominância pela arcada superior e maior prevalência em incisivos laterais, podendo ocorrer bilateralmente (HULSMANN, 1997; DE ROSSI et al., 2013; HEYDARI; RAHMANI, 2015).

De acordo com a classificação de Oehlers, existem três tipos de invaginações, classificadas conforme a sua profundidade e complexidade. São elas: a) tipo I, uma invaginação que ocorre dentro dos limites da coroa, o esmalte fica enclausurado na coroa e, portanto, não se estende até a junção cemento-esmalte; b) tipo II, uma invaginação revestida de esmalte que vai além da junção cemento-esmalte, formando um saco cego dentro da raiz, que pode ou não se comunicar com a polpa dentária e não se estende aos tecidos periodontais; c) tipo III, apresenta uma invaginação que se aprofunda na raiz e forma um forame adicional e comumente não está conectada à polpa (OEHLERS, 1957).

Diante disso, o objetivo deste trabalho é abordar sobre as diferentes intervenções clínicas descritas na literatura, com foco na necessidade de tratamento endodôntico nos casos de dens in dente conforme sua classificação.

\section{METODOLOGIA}

Realizou-se uma busca nas bases de dados PubMed, Biblioteca Virtual da Saúde (BVS) e Google Acadêmico utilizando as palavras-chaves "Dens in dente", "Dens invaginatus", "dental malformation", "endodontic treatment", "root canal treatment", "endodontic therapy", "endodontics" combinadas com os operadores booleanos "AND" e "OR".

Foram incluídos artigos publicados no período de 1980 a 2017, nos idiomas Português, Inglês e Espanhol, que abordassem o tratamento realizado em Dens in dente dos tipos I, II e III. Foram excluídos artigos que não fizessem referência ao tratamento realizado ou que abordassem outro assunto. No total, foram selecionados 27 artigos para análise e revisão.

\section{DESENVOLVIMENTO}

Diversos trabalhos têm sido publicados apresentando casos clínicos de Dens in dente com diferentes tipos de tratamento, sendo a abordagem selecionada de acordo com a complexa anatomia. Cada conformação de Dens in dente tem sua particularidade, e as intervenções podem ser de várias formas, que incluem tratamento preventivo, como selamento do esmalte (OZBAS; SUBAY; ORDULU, 2010), tratamento endodôntico convencional (SOUZA; BRAMANTE, 1998), tratamento cirúrgico combinado (ISHIDA et al., 2016), reimplante intencional (HULSMANN, 1997) e extração (SOUZA; BRAMANTE, 1998). 
A preservação da estrutura dentária é um dos objetivos do tratamento endodôntico. O tipo I geralmente não necessita da intervenção endodôntica pois está restrito em esmalte, assim, pode ser realizado um selamento da abertura da coroa com material restaurador para evitar possíveis comunicações com a polpa (OZBAS; SUBAY; ORDULU, 2010) e realizar um acompanhamento clínico. Souza, Tavano e Bramante (1999) realizaram estudos onde foi executado a profilaxia e posteriormente a confecção do selante em dens in dente do tipo I.

Os casos tipo II podem apresentar um saco cego dentro da raiz e haver ou não comunicação com a polpa, sendo a escolha do tratamento de acordo com a situação presente. Após o irrompimento do dente, a invaginação pode se comunicar com a cavidade oral e os tecidos do lúmen do espaço interno se tornarem necrosado. A maioria desses dentes passa pelo processo de tratamento endodôntico por se encontrarem com tecido contaminado no momento do diagnóstico (HULSMANN, 1997). Os tratamentos que podem ser realizados para o tipo II são tratamento endodôntico convencional e tratamento endodôntico associado ao cirúrgico (SOUZA; BRAMANTE, 1998; ISHIDA et al., 2016). É relatado na literatura que no passado o tratamento era limitado a intervenção cirúrgica ou a extração (SATHORN; PARASHOS, 2007; KELES; ÇAKICI, 2010).

No tipo III, o tratamento também é indicado conforme a situação presente, no entanto, a terapia endodôntica convencional normalmente é realizada devido à proximidade ou comunicação da invaginação com o tecido pulpar. Tsurumachi (2004) relatou um caso clínico onde apenas o canal invaginado foi tratado mantendo a vitalidade pulpar. O dente apresentava-se com lesão perirradicular comunicado ao canal invaginado. $\mathrm{O}$ tratamento endodôntico foi suficiente para reparação da lesão e a saúde pulpar foi preservada. O tratamento endodôntico apenas da invaginação de dens in dente tipo III também foi relatado por Fristad e Molven (1998) e Ford (1998). Hulsmann (1997) afirmou que se o tecido pulpar não tiver comunicação com espaço do lúmen, o tratamento apenas da invaginação é suficiente nos casos tipo III e um acompanhamento desses dentes deve ser realizado para verificar a manutenção da vitalidade pulpar e a reparação periapical, porém se há relação entre lúmen e o tecido pulpar ambos devem ser tratados, podendo ser de forma independente ou unir toda estrutura como se fosse uma cavidade única (PRADEEP et al., 2012; MARTINS et al., 2016).

$\mathrm{O}$ acesso à invaginação pode ser obtido com brocas ou insertos ultrassônicos e, para um aumento na capacidade visual, o microscópio operatório pode ser utilizado no atendimento clínico. Para Souza e Bramante (1998) a primeira dificuldade encontrada é a obtenção do acesso adequado ao canal devido à possibilidade de perfurações, tornando essencial a realização de radiografias em três ângulos horizontais para prevenir perfurações radiculares. A literatura enfatiza que a tomografia computadorizada de feixe cônico (TCFC) pode ser utilizada como técnica radiológica de escolha, pois permite a observação em diferentes planos e fornece ao clínico uma imagem tridimensional (PRADEEP et al., 2012), assim como relatado por Ishida et al. (2016), que utilizaram a tomografia de feixe cônico no diagnóstico de um dens in dente tipo II e por Ranganathan, Sundaresan e Ramasamy (2016), em um caso tipo III.

O processo de limpeza e modelagem, assim como a obturação desses dentes, geralmente são de difícil execução técnica devido à complexidade da anatomia interna, que facilita a permanência de bactérias e resíduos de polpa necrótica dificultando o acesso dos instrumentos nessas regiões, e requerem modificações na técnica de tratamento e cuidados adicionais (SKONER; WALLACE, 1994; SOUZA; BRAMANTE 1998; ISHIDA et al., 2016).

O hipoclorito de sódio tem sido a substância química auxiliar de escolha na maioria dos trabalhos analisados. Nos trabalhos de Pradeep et al. (2012), Subbiya et al. (2013) e Martins et al. (2016), a solução irrigadora de hipoclorito de sódio utilizada foi na porcentagem de 5,25\%; a concentração de 2,5\% utilizada nos trabalhos de De Rossi et al. (2013), Heydari e Rahmani (2015), Ishida et al. (2016) e Ranganathan, Sundaresan e Ramasamy (2016) e a concentração de 1,5\% foi no relato de Pinto et al. (2017). Em casos de ápice aberto assim como em um de seus relatos Martins et al. (2016) utilizou a clorexidina a $2 \%$ por conta do tamanho da abertura apical, sendo 
contraindicado o hipoclorito de sódio devido à possibilidade de causar danos aos tecidos periapicais. No processo químico-mecânico de limpeza e irrigação alguns autores como Skoner e Wallace (1994) e Souza e Bramante (1998) recomendam fazer o uso de instrumentação ultrassônica, a qual tem apresentado resultados eficazes.

Após o preparo químico mecânico, pode ser utilizada a medicação intracanal a base de hidróxido de cálcio quando o tratamento endodôntico não pode ser concluído numa mesma sessão. Nos casos de dens in dente tipo II e III em dentes permanentes com rizogênese incompleta e diagnosticados com necrose pulpar associados a alterações periapicais agudas ou crônicas, é necessário estimular a formação de uma barreira no ápice radicular pelo método da apicificação com hidróxido de cálcio. Ferguson, Friedman e Frazzetto (1980) relataram um caso de apicificação em dens in dente, no qual foram realizadas trocas de hidróxido de cálcio durante um ano e, após fechamento apical, realizada a obturação do canal com guta-percha, concluindo o tratamento com sucesso. Portanto, se o tratamento obteve a formação da barreira apical e a reparação tecidual, podese concluir a terapia endodôntica com o uso da técnica obturadora da guta-percha termoplastificada (HULSMANN, 1997; LUCAS; LUCAS, 2003; SILVA et al., 2011). Nas situações em que o tratamento conservador não foi eficaz, torna-se necessário realizar a cirurgia parendodôntica com preparo retrógrado (GHARECHAHI; GHODDUSI, 2012).

As técnicas de obturação mais utilizadas e indicadas são a termocompactação e a injeção termoplastificada (SOUZA; BRAMANTE, 1998; LUCAS; LUCAS, 2003; SILVA et al., 2011; ISHIDA et al., 2016; RANGANATHAN; SUNDARESAN; RAMASAMY, 2016). Para Rotstein et al. (1987) a técnica de condensação vertical também é indicada. A condensação lateral foi relatada Ferguson, Friedman e Frazzetto (1980) e Rani e Sroa (2015).

A cirurgia parendodôntica com preparo retrógrado é realizada quando o tratamento endodôntico convencional não obteve êxito devido à complexidade do sistema de canais e a associação de técnicas geralmente é realizada quando há permanência de sintomatologia e/ou lesão periapical e fístula após o elemento dentário ter sido submetido ao tratamento não cirúrgico (TSURUMACHI; HAYASHI; TAKEICHI, 2002). Alguns autores, como Hulsmann (1997) e Tsurumachi, Hayashi e Takeichi (2002), concordam que a cirurgia pode ser realizada em situações onde não há possibilidades do tratamento convencional devido à anatomia complexa, incapacitando o acesso aos canais assim como relatado por Sauveur, Sobel e Boucher (1997).

O reimplante intencional, um tratamento cirúrgico que consiste na exodontia, realização do procedimento extraoral e reimplante no alvéolo, é um recurso utilizado apenas quando os tratamentos conservador e cirúrgico associados falham (HULSMANN, 1997). Lindner, Messer e Tyas (1995) relataram um caso em que o reimplante intencional foi realizado utilizando esta modalidade de tratamento, o qual mostrou uma reparação tecidual bem sucedida da lesão periapical. $\mathrm{Na}$ literatura pouco se encontra a respeito de reimplante intencional em dens in dente.

A extração é indicada em dentes com irregularidades anatômicas ou então dentes supranumerários que impossibilitem a realização de tratamentos convencionais ou cirúrgicos (HULSMANN, 1997). Para Rotstein et al. (1987) a extração pode ser indicada em casos de estrutura anormal da coroa, com problemas estéticos e/ou funcionais.

Uma nova alternativa foi relatada por Pinto et al. (2017) em um caso de dens in dente tipo II, no qual foi utilizado Fibrina Leucocitária Rica em Plaquetas para estimular formação apical e promover cicatrização da lesão óssea.

O diagnóstico do dens in dente pode ser realizado pela associação do exame clínico ao exame de imagem, especialmente com o uso da tomografia computadorizada de feixe cônico (TCFC), uma ferramenta de grande importância no diagnóstico endodôntico por permitir uma visualização tridimensional dos tecidos e alterações dentárias e periapicais (DE ROSSI et al., 2013; ISHIDA et al., 2016; RANGANATHAN, SUNDARESAN; RAMASAMY, 2016), sendo descobertos muitas vezes em consultas de rotina (HULSMANN, 1997). Durante o exame intrabucal, o paciente pode apresentar edema, inchaço ou estar com aspecto de normalidade. Quanto à sintomatologia, pode apresentar-se positiva ou negativa. Para um eficaz diagnóstico, alguns testes 
clínicos como inspeção, percussão, palpação e testes térmicos podem nos auxiliarem na obtenção do diagnóstico (HULSMANN, 1997; SOUZA; BRAMANTE, 1998).

Devido à invaginação do esmalte, os dentes acometidos podem apresentar em sua estrutura uma profunda fossa lingual no local onde ocorre a invaginação (NEVILLE et al., 2009). O tratamento mais indicado e a necessidade de terapia endodôntica dependerão do tipo de invaginação e das características clínicas apresentadas por esse dente. A intervenção endodôntica em casos de malformação como o dens in dente requer do profissional o conhecimento da anatomia interna do dente a ser tratado, que muitas vezes apresenta um maior grau de complexidade (HULSSMAN, 1997; SOUZA; BRAMANTE, 1998).

O preparo químico-mecânico possui um papel importante durante a fase da terapia endodôntica, sendo assim a etapa de desinfecção dos canais radiculares determina o sucesso da terapia endodôntica. O hipoclorito de sódio parece ser a substância química auxiliar mais vantajosa devido às suas propriedades antimicrobianas e capacidade de dissolução tecidual. Quanto a concentração, maiores concentrações parecem denotar maior efeito antibacteriano, porém não existe um consenso na literatura de qual concentração deve ser utilizada. Para Sim et al. (2001), a maioria dos dentistas americanos faz o uso de hipoclorito de sódio a 5,25\%. Clinicamente, as concentrações entre 0,5\% e 6\% são recomendadas (ZEHNDER, 2006; ROSSI et al., 2013; HEYDARI; RAHMANI, 2015; ISHIDA et al., 2016; RANGANATHAN; SUNDARESAN; RAMASAMY, 2016). A solução irrigadora deve ser renovada constantemente durante o processo da instrumentação e utilizada durante todo o processo de preparo dos canais.

O uso do ultrassom foi recomendado por Skoner e Wallace (1994); Souza e Bramante (1998) e Martins et al. (2016) com a finalidade de auxiliar na limpeza do canal radicular, uma vez que a agitação da solução irrigadora com insertos ultrassônicos auxilia na dissolução dos tecidos (VAN DER SLUIS et al., 2010), na remoção de detritos e resíduos e na penetração da solução para que essa atinja áreas de complexidades anatômica que o instrumento endodôntico não toca.

O Ácido Etilenodiamino Tetracético Dissódico (EDTA) é um material usado para remoção da smear layer. Malheiros, Marques e Gavine (2005) apontam que a smear layer aglomera em sua composição os microrganismos, podendo ser uma das causas do insucesso endodôntico. O EDTA é utilizado após a instrumentação do canal, promovendo a limpeza das paredes dentinárias, aumento na permeabilidade por reagir com os íons cálcio da dentina, removendo-os e possibilitando que a medicação intracanal alcance os lugares onde a smear layer poderia impedir (SEN; WESSELINK; TURKUN, 1995). O agente quelante atua na matéria inorgânica da dentina, tornando necessário fazer seu uso com a combinação do hipoclorito de sódio, o qual age no componente orgânico da dentina (TANEJA; KUMARI; ANAND, 2014). Deve ser utilizado antes da medicação intracanal e/ou antes da obturação dos canais radiculares.

O hidróxido de cálcio é a medicação intracanal mais utilizada devido suas propriedades antimicrobianas e biocompatibilidade (SOUZA; BRAMANTE, 1998; SIQUEIRA JUNIOR; LOPES, 1999). O hidróxido de cálcio pode ser utilizado com veículos aquosos, como o soro fisiológico, ou viscosos, como o propilenoglicol, e é indicado quando o tratamento não pode ser concluído em uma sessão, ou em casos de rizogênese incompleta na técnica de apicificação, um método não cirúrgico com o objetivo de formação de um tecido mineralizado no ápice radicular resultando em um tampão apical. O material induz a formação de uma área mineralizada e o fechamento apical, pela deposição de tecido duro que poderá ser visível radiograficamente (SEGUI et al., 2013). Além do hidróxido de cálcio, o agregado de trióxido mineral (MTA) também é indicado nos casos de rizogênese incompleta com a finalidade de confecção de um tampão apical, por apresentar resultados satisfatórios (SILVA et al., 2011; GHARECHAHI; GHODDUSI, 2012). Em ambos os casos, após o selamento apical, procede-se com a obturação do canal radicular (HÜLSMANN, 1997; LUCAS; LUCAS, 2003; SILVA et al., 2011).

A técnica de obturação dos canais mais relatada na literatura foi a termoplastificada (SOUZA; BRAMANTE, 1998; LUCAS; LUCAS, 2003; SILVA et al., 2011; ISHIDA et al., 2016), 
sendo seu uso justificado pelo escoamento da guta-percha plastificada para o interior dos locais de difícil acesso (SOUZA; BRAMANTE, 1998).

Tratamento endodôntico associado com o cirúrgico pode ser uma das opções para dens in dente tipos II e III quando o tratamento endodôntico sozinho não foi o suficiente para eliminar o agente etiológico (HULSMANN, 1997; TSURUMACHI; HAYASHI; TAKEICHI, 2002). A cirurgia parendodôntica tem o objetivo principal de promover a remoção do processo patológico periapical a fim de dar condições para que o reparo dos tecidos aconteça (NISHIYAMA; LEAL; CAMARGO, 2002). O reimplante intencional e a extração são recursos pouco utilizados, ficando restritos à dentes que não podem receber tratamento via canal convencional ou cirúrgico. $\mathrm{O}$ reimplante consiste na técnica de remoção do dente do alvéolo, com trauma mínimo a essas duas estruturas e, após realizado o tratamento, sua reinserção. A extração é indicada para situações que as outras modalidades cirúrgicas não obtiveram sucesso ou não são indicadas, dentes com problemas estéticos e/ou funcionais e dentes supranumerários (ROTSTEIN et al., 1987; HULSMANN, 1997).

Com o crescimento dos estudos, novas alternativas estão sendo estudadas para o tratamento para o dens in dente, como o procedimento endodôntico regenerativo associado à cirurgia parendodôntica, indicados para dentes com formação incompleta de raiz e lesões apicais de grande extensão, como relatado por Pinto et al. (2017). Pode-se observar por meio de TCFC que fechamento apical e regeneração periapical.

\section{CONCLUSÃO}

Por meio dessa revisão da literatura, pode-se observar que o tratamento dos casos de dens in dente mostrou-se complexo devido à diversidade de suas apresentações clínicas. A abordagem dos casos de dens in dente requer do profissional conhecimento sobre diagnóstico, interpretação radiográfica e tomográfica, conhecimento das possibilidades de intervenção clínica de acordo com a sua classificação e domínio de anatomia interna. Embora existam muitos artigos na literatura que apresentem o tratamento dos casos dessa malformação, as descrições dos protocolos clínicos utilizados são falhas e com poucas informações, dificultando a reprodução e a disseminação deste assunto.

\section{REFERÊNCIAS}

ALANI, A.; BISHOP, K. Dens invaginatus. Part 1: classification, prevalence and aetiology. International Endodontic Journal, v. 41, n. 12, p. 1123-1136, 2008.

FERGUSON, F. S.; FRIEDMAN, S.; FRAZZETTO, V. Successful apexification technique in an immature tooth with dens in dente. Oral Surgery, Oral Medicine, Oral Pathology and Oral Radiology, v. 49, n. 4, p. 356-459, 1980.

FORD, H. E. P. Peri- radicular inflammation related to dens invaginatus treated without damaging the dental pulp: a case report. International Journal of Paediatric Dentistry, v. 8, n. 4, p. 283286, 1998.

FRISTAD, I.; MOLVEN, O. Root resorption and apical breakdown during orthodontic treatment of a maxillary lateral incisor with dens invaginatus. Dental Traumatology, v. 14, n. 5, p. 241-244, 1998.

GHARECHAHI, M.; GHODDUSI, J. A nonsurgical endodontic treatment in open-apex and immature teeth affected by dens invaginatus: using a collagen membrane as an apical barrier. The Journal of the American Dental Association, v. 142, n. 2, p. 144-148, 2012. 
HEYDARI, A.; RAHMANI, M. Treatment of dens invagination in a maxillary lateral incisor: A case report. Iranian Endodontic Journal, v. 10, n. 3, p. 207-209, 2015.

HULSMANN, M. Dens invaginatus: etiology, classification, prevalence, diagnosis, and treatment considerations. International Endodontic Journal, v. 30, n. 2, p. 79-90, 1997.

ISHIDA, A. L. et al. Treatment of extensive cystic lesion in the maxilla associated with dens in dente. Brazilian Dental Science, v. 19, n. 3, p. 117-123, 2016.

KARACA, I.; TOLLER, M. Ö. Multiple bilateral dens in dente involving all the premolars. Case report. Australian Dental Journal, v. 37, n. 6, p. 449-452, 1992.

KELES, A.; ÇAKICI, F. Endodontic treatment of a maxillary lateral incisor with vital pulp, periradicular lesion and type III dens invaginatus: a case report. International Endodontic Journal, v. 43, n. 7, p. 608-614, 2010.

KIRZIOGLU, Z.; CEYHAN, D. The prevalence of anterior teeth with dens invaginatus in the western Mediterranean region of Turkey. International Endodontic Journal, v. 42, n. 8, p. 727 734, 2009.

LINDNER, C.; MESSER, H. H.; TYAS, M. J. A complex treatment of dens invaginatus. Dental Traumatology, v. 11, n. 3, p. 153-155, 1995.

LUCAS, G. V; LUCAS, O. N. Dens invaginatus: tratamiento en un diente permanente joven. Revista de la Asociación Dental Mexicana, v. 60, n. 6, p. 229-232, 2003.

MALHEIROS, C. F.; MARQUES, M. M.; GAVINE, G. In vitro evaluation of the cytotoxic effects of acid solutions used as canal irrigants. Journal of Endodontics, v. 31, n. 10, p. 746-748, 2005.

MARTINS, J. N. et al. Endodontic management of dens invaginatus Type IIIb: Case series. European Journal of Dentistry, v. 10, n. 4, p. 561-565, 2016.

NEVILLE, B. W. et al. Patologia Oral e Maxilofacial. 3. ed. Rio de Janeiro: Elsevier, p. 89-91, 2009.

NISHIYAMA, C. K.; LEAL, J. M.; CAMARGO, J. M. P. Tratamento Cirúrgico das Infecções Periapicais: Quando Indicar. In: CARDOSO, R. J. A; GONÇALVES, E. A. N. Odontologia: Arte, Ciência e Técnica. Endodontia, Trauma. São Paulo: Artes Médicas, v. 2, p. 369-390, 2002.

OEHLERS, F. A. C. Dens invaginatus (dilated composite odontome): I. Variations of the invagination process and associated anterior crown forms. Oral Surgery, Oral Medicine and Oral Pathology, v. 10, n. 11, p. 1202-1218, 1957.

OZBAS, H.; SUBAY, R. K.; ORDULU, M. Surgical retreatment of an invaginated maxillary central incisor following overfilled endodontic treatment: a case report. European Journal of Dentistry, v. 4, n. 3, p. 324-328, 2010.

PINTO, N. et al. An innovative regenerative endodontic procedure using leukocyte and platelet-rich fibrin associated with apical surgery: A Case Report. Journal of Endodontics, v. 43, n. 11, p. 1828-1834, 2017. 
PRADEEP, K. et al. Conservative management of type III dens in dente using cone beam computed tomography. Journal of Clinical Imaging Science, v. 2, n. 51, p. 1-5, 2012.

RANGANATHAN, J.; SUNDARESAN, M. K. R.; RAMASAMY, R. Management of Oehler's type III Dens Invaginatus using cone beam computed tomography. Case Reports in Dentistry, v. 2016, n. 6, p. 1-6, 2016.

RANI, N.; SROA, R. B. Nonsurgical endodontic management of dens invaginatus with open apex: A case report. Journal of Conservative Dentistry, v. 18, n. 6, p. 492-495, 2015.

ROSSI, A. et al. The treatment of a maxillary lateral incisor with unusual morphology with long- term follow- up. International Endodontic Journal, v. 46, n. 12, p. 1183-1190, 2013.

ROTSTEIN I. et al. Clinical considerations in the treatment of dens invaginatus. Endodontics \& Dental Traumatology, v. 3, n. 5, p. 249-254, 1987.

SATHORN, C.; PARASHOS, P. Contemporary treatment of class II dens invaginatus. International Endodontic Journal, v. 40, n. 4, p. 308-316, 2007.

SAUVEUR, G.; SOBEL, M.; BOUCHER, Y. Surgical treatment of a lateroradicular lesion on an invaginated lateral incisor (dens in dente). Oral Surgery, Oral Medicine, Oral Pathology, Oral Radiology and Endodontics, v. 83, n. 6, p. 703-706, 1997.

SEGUI, R. et al. Root Fortification. Journal of Endodontics, v. 39, n. 6, p. 57-62, 2013.

SEN, B. H.; WESSELINK, P. R.; TURKUN, M. The smear layer: a phenomenon in root canal therapy. International Endodontic Journal, v. 28, n. 3, p. 141-148, 1995.

SERRANO, J. Triple dens invaginatus in a mesiodens. Oral Surgery, Oral Medicine, Oral Pathology and Oral Radiology, v. 71, n. 5, p. 648-649, 1991.

SILVA, F. W. G. P. et al. Root canal treatment of an immature dens invaginatus with apical periodontitis: a case report. Journal of Dental for Children, v. 78, n. 1, p. 66-70, 2011.

SIM, T. P. C. et al. Effect of sodium hypochlorite on mechanical properties of dentine and tooth surface strain. International Endodontic Journal, v. 34, n. 2, p. 120-132, 2001.

SIQUEIRA JUNIOR, J. F.; LOPES, H. P. Mechanisms of antimicrobial activity of calcium hydroxide: a critical review. International Endodontic Journal, v. 32, n. 5, p. 361-369, 1999.

SKONER, J. R.; WALLACE, J. A. Dens invaginatus: another use for the ultrasonic. Journal of Endodontics, v. 20, n. 3, p. 138-140, 1994.

SOUZA, S. M. G.; BRAMANTE, C. M. Dens invaginatus: treatment choices. Endodontics \& Dental Traumatology, v. 14, n. 4, p. 152-158, 1998.

SOUZA, S. M. G.; TAVANO, S. M.; BRAMANTE, C. M. Unusual case of bilateral talon cusp associated with dens invaginatus. International Endodontic Journal, v. 32, n. 6, p. 494-498, 1999. 
SUBBIYA, A. et al. An unusual type II dens invaginatus with calcified canals in a maxillary lateral incisor-a case report. Journal of Clinical and Diagnostic Research, v. 7, n. 9, p. 2084-2085, 2013.

TANEJA, S.; KUMARI, M.; ANAND, S. Effect of QMix, peracetic acid and ethylenediaminetetraacetic acid on calcium loss and microhardness of root dentine. Journal of Conservative Dentistry, v. 17, n. 2, p. 255-158, 2017.

TSURUMACHI, T. Endodontic treatment of an invaginated maxillary lateral incisor with a periradicular lesion and a healthy pulp. International Endodontic Journal, v. 37, n. 10, p. 717$723,2004$.

TSURUMACHI, T.; HAYASHI, M.; TAKEICHI, O. Non-surgical root canal treatment of dens invaginatus type 2 in a maxillary lateral incisor. International Endodontic Journal, v. 35, n. 1, p. 68-72, 2002.

VAN DER SLUIS, L. W. et al. Study on the influence of refreshment/activation cycles and irrigants on mechanical cleaning efficiency during ultrasonic activation of the irrigant. Journal of Endodontics, v. 36, n. 4, p. 737-740, 2010.

ZEHNDER, M. Root canal irrigants. Journal of Endodontics, v. 32, n. 5, p. 389-398, 2006. 